\title{
Observations on Time 2012 REMADE
}

\author{
SaiPrahlad K
}

\begin{abstract}
The purpose of this paper is to present author's observation. The main topics in the paper are force, motion and time. All three (force, motion and time) are interconnected to space and energy. The commonly used term 'Spacetime' is an observation given to us by Einstein's general relativity; Kindly note: it does not mean 'time' is a separate dimension. What spacetime really means is that time is related to gravity. We alreadyknow that [1] gravity is caused by mass(which is energy $E=M C 2$ ) and[2] gravity is the curvature of space. Therefore, time is not only related to space but also related to energy. This paper connects General relativity with thermodynamics, and it provides a solution to what causes force, motion and time.
\end{abstract}

\section{Introduction:}

Time is best observed by change, a change in physical or chemical process.

What is change? And what causes change?

From a scientific perspective, Change is caused by a Force. Example: The Electromagnetic force, the gravitational force, the strong nuclear force and the weak nuclear force. A change in position of an object is Motion; it can also be described as velocity, acceleration, displacement and time. Motion is observed by attaching a frame of reference to a body and measuring its change in position relative to another reference. As there is no absolute frame of reference absolute motion cannot be determined. Everything in the universe can be considered to be moving (in a state of motion). An object's motion cannot change unless it is acted upon by a force.

Force Causes -> Motion -> motion causes -> Change

In reality, motion and change are the same.

\section{Observations from our everyday lives:}

\section{Method:}

\section{Observation A}

1. Take a clock

2. Observe the changes happening in the clock, and note the passing of time.

3. Time period 15 seconds to 1 hour.

\section{Observation B}

1. Take an electronic gadget, preferably a laptop or a mobile phone

2. Keep the device on, till it runs out of battery

3. Note the changes and causes that lead the gadget to run out of electricity

Observation C

1. Take the sun

2. Observe its changes and note down the process

3. Time period for 2 days or a week.

\section{Observation D}

1. Take a living organism, preferably ourselves

2. Observe the changes that are happening in a day, and ask your-self the question why do we need food, water and oxygen for survival? What is the reason?

\section{Observation E}

1. Take the Human eyes (not literally) or any photoreceptors, including telescopes, Binoculars etc.

2. Observe and ask the question: why are we able to see? What is the process?

3. Note down your conclusions

Observation F (for physicists)

1. Take the Effect of Gravitational time dilation

2. Write down your observations on why and how gravity affects time

3. Note the changes that are accruing in the object you're observing in differing gravitational potentials. Example: An object inside a black hole or above earth's atmosphere etc., 


\section{Author's results and Discussions:}

\section{Observation A}

When time passes: energy is released from the clock, by the means of sound (tick, tick, tick) and as photons (thermal radiation). This release of energy is the change that was happening in the clock when time was flowing forward.

\section{Observation B}

When time passes: Energy is being released into the universe (from the laptop). The energy that was released \radiated away from the laptop is thermal radiation, photons, and sound. Due to this constant release of energy the laptop ran out of battery. The same is true for any electronic gadget. And this is also why we need constant supply of electricity for our cool gadgets and luxury items to perform work.

\section{Observation C}

When time passeslwhen time flows forward: energy is being released, in this case from the sun to the universe. Note: Energy is released whenever an object performs an action or work. Stars perform work 24/7 till the end of their lives. Therefore, it is no coincidence that stars too release energy constantly in the universe; and it is only because of this constant release of energy we are able to receive the sunlight, continuously. If energy is released randomly then the sunlight we receive here on earth will also be random. Known Facts: A) The sun loses 1 billion kilograms (2.2 billion pounds) of weight each second; B) Enough energy reaches the earth from the sun every second to fulfill all our power needs for a year.

\section{Observation D}

A living organism is like a machine. It cannot stay idle, if it is alive. The Human body for example performs work continuously just like our sun. An adult human takes about 23,000 breaths per day. Our Heart beats about 35 million times a year. Our brain receives about 100 million pieces of information at any one moment from your eyes, nose, ears, skin and receptors inside our body. The amount of electrical energy generated by our brain is enough to power a light bulb! Messages are sent to our brain at around 360 kilometers (224 miles) per hour. Our Stomach lining replaces itself every three days. Our body destroys or loses two and a half million red blood cells every second. Luckily, it creates more at the same rate! In one day, our blood travels 19,312 Kilometers (12,000 miles)! We release energy constantly. Therefore we need food, water and oxygen to survive (conservation Law).

\section{Observation E}

The Human eye is a complex optical system which collects light from the surrounding environment, regulates its intensity through a diaphragm, focuses it through an adjustable assembly of lenses to form an image, converts this image into a set of electrical signals, and transmits these signals to the brain through complex neural pathways that connect the eye via the optic nerve to the visual cortex and other areas of the brain.

This complex process so easily done by our body works because energy is released constantly from our surroundings. Example and fact: Think of a light beam. A light beam in a billionth of a second travels about a foot. Now, when you look at yourself in a mirror in the morning, you say to yourself, well that's me! Actually that is not you at all. You are looking at an image that was you a billionth of a second ago; because that is the time it takes for light to go from your face to the mirror and back to your eyes. Same thing for the moon; when you look at the moon you are not really looking at the moon as it is at all, you are looking at the moon as it was about a second ago.

If energy was not released from our surroundings: then the whole universe would become a perfect black body, like a black hole (invisible) but without gravity effects (note: this is only a description). Fact with author's results: Information inside black holes is invisible to us because energy is not released from blackholes.

\section{Observation F}

Gravitational time dilation: It is the effect of time passing at different rates in regions of different gravitational potential; the lower the gravitational potential, the more slowly time passes. Albert Einstein originally predicted this effect in his theory of relativity and it has since been confirmed by test of general relativity. This has been demonstrated by noting that atomic clocks at differing altitudes (and thus different gravitational potential) will eventually show different times. The effects detected in such experiments are extremely small, with differences being measured in nanoseconds.

The Reason atomic clocks show different times in differing altitudes is because of gravity and the release of energy. When the gravitational potential is low, energy is released at a slower rate, slowing down time. Example: Living in a planet. When the gravitational potential is high, energy is released at a faster rate, increasing time. Example: Living in a space station or zero $\mathrm{G}$ environments. 


\section{This Release of energy from everything, does it exist? And is there proof?}

It does exist and it is already a known scientific fact, but the information was perceived differently. Two main facts:

1. Thermal Radiation (includes Black body Radiation)

2. Transformation of Energy

\section{Thermal Radiation:}

All matter above absolute zero degree temperature releases thermal radiation. According to the third law of thermodynamics, it is impossible to reach absolute zero degree temperature, because a minimum amount of entropy (heat) always exists. Therefore all matter and antimatter present in the universe release energy (thermal radiation).

\section{Black body Radiation:}

A perfect black body is a body that absorbs and stores information, indefinitely. As there is no perfect black bodies present in the universe: everything matter and antimatter release energy. This release of energy is commonly called as black body radiation.

\section{Transformation of Energy:}

Transformation of energy is literally transformation of energy. We see it and use it every day and in everything; it is the core of physics. The conservation law is based on it. Examples: 1 . When we push an object (apply force) we transfer energy from A to B, chemical to physical. 2. Eyesight: Photons emitted from our environment is absorbed by our eyes and converted into electrical and neurological (chemical) signals (transformation of energy). Other examples: Workings of Broadband, DVD, etc. Release of energy and transformation of energy is the same, but only when energy is released it can be transferred. These facts are the proof to author's claim.

\section{Observation X}

\section{So Far we know:}

1. Energy is released constantly in the universe.

2. Release of energy (\&transformation of energy) causes change to a physical or to a chemical system; Change results to time. Release of energy causes "Change", which is "Time".

3. Release of energy (or transformation of energy) can also be described as Force

4. If energy is not released into the universe: the object becomes like a black hole (invisible) but without gravity effects. Ceasing all physical and chemical process to take place.

In this observation $\mathrm{X}$, we are going to look at the possibility if the release of energy can be reversed in a given system. Let us take the observations $\mathrm{ABC}$ and $\mathrm{E}$. Before we begin, you must know that whenever energy is released into the cosmos the process makes an impact. The impact is usually information stored by an observer. So trying to reverse an impact event could destroy the information stored by the observer.

A. If we reverse the energy releases that accrued in a clock. Then the clock would gain its energy back. It is like time travellthe decrease of entropy in a system.

B. Likewise, the laptop too will gain its energy back

C. The sun would become young and heavy, the fusion processes will be reversed,

D. (From Observation E) We would forget events because the photons are never absorbed in the first place.

Observation $\mathrm{X}$ is only hypothetical because it is impossible for us to decrease entropy in a system. Even if we did decrease entropy in one particular area of space, the entropy outside that space will compensate for it, literally making us impossible to time travel or decrease entropy.

\section{Conclusion:}

Based on all the observations and facts, the author deduces that "Release of energy and transformation of energy is time", more accurately "rate at which energy is released defines time", becauserelease of energy and transformation of energy causes change to a system and this creates the flowlarrow of time. But this definition leads us to a new question: 'what causes energy to be released and transferred?' For this the author hypothesizes the following: Big bang theory states that everything (all matter and antimatter; which is pure energy) was contained in volume less than one trillionth the size of a point of a pin, meaning the size of an atom. From that point, the universe expanded to its present state. There are many theories to explain this expansion of the universe; personally author prefers dark energy as the culprit, and furthermore, he proposes dark energy as an opposite form of purelordinary energy. He arrives at this conclusion based on two facts: 1. Third law of thermodynamics which state that all matter and antimatter have heat, and 2. Universe is not in thermodynamic equilibrium i.e. temperature of the universe defers from place to place. If the universe had only matter and no 
space then such a universe would be unchangeable, in a state of complete equilibrium. But if the universe had space and matter (energy) then such a universe would be in constant change, in a state of chaos and difference. Our universe has energy (matter) and space. Therefore, it is constantly changing. Space is like dark energy without it the universe would not have expanded, but because of it, the universe is still expanding. Since, everything we see and everything we are, is made up of matter ( $\mathrm{E}=\mathrm{MC} 2)$, energy is constantly being radiated away and this constant release of energy gives rise to Force, motion and time. Recap:

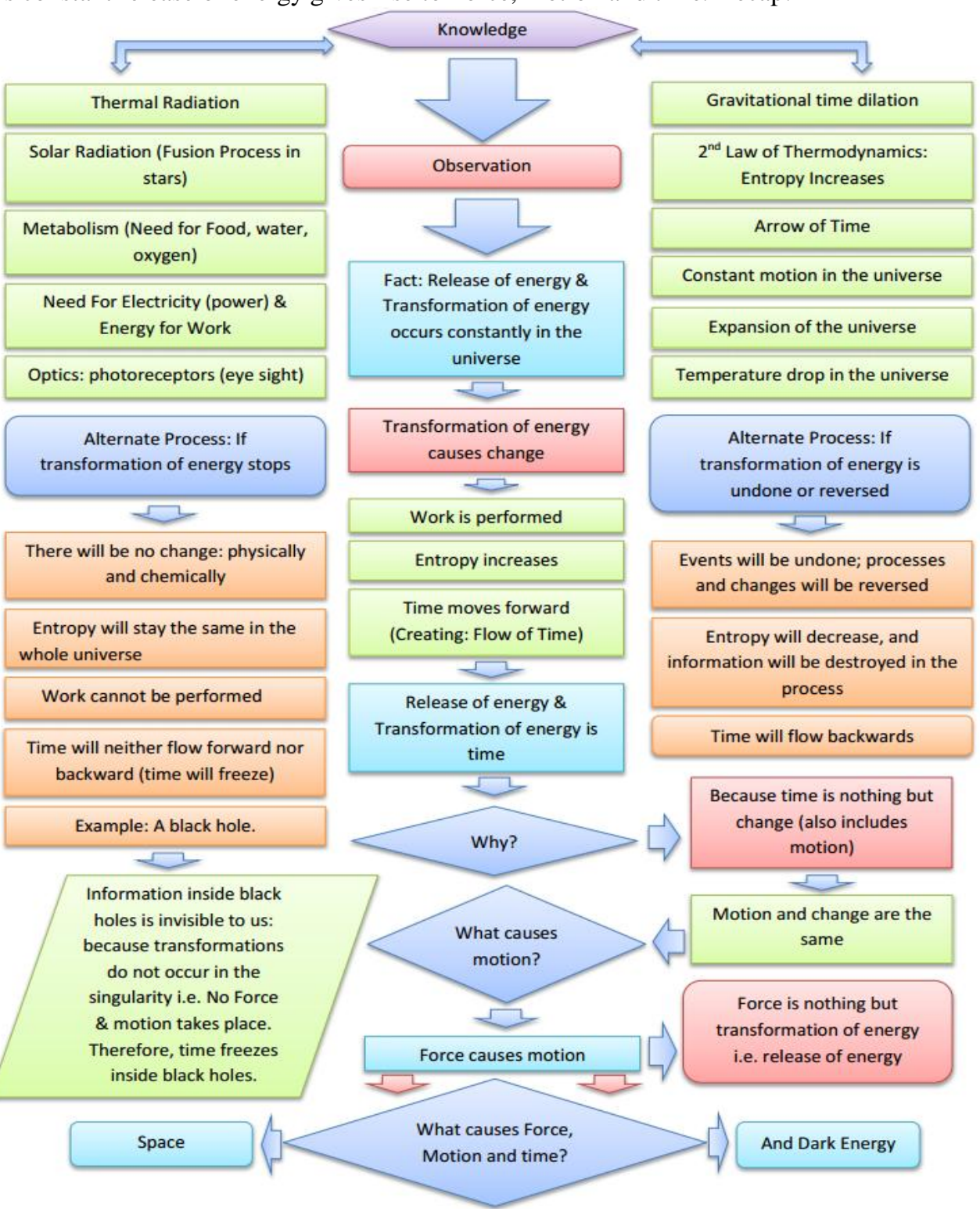

FAQs:

1. What is time?

Answer: It is motion, or any change.

2. How is time created?

Answer: By force.

3. What triggered time in the early universe, at the big bang?

Answer: Empty space and dark energy

4. What causes force, motion and time?

Answer: Space and Dark energy

5. Do photons have time?

Answer: For photons time is infinite. Photons don't stay at one place, they mingle, and merge and spread like a wave in the entire cosmos. They are always in motion, so is everything in the cosmos. Every particle is a wave, the duality.

6. What causes this motion?

Answer: Classical answer force. Modern answer: Dark energy and empty space. 
7. What happens to an object which releases energy constantly?

Answer: The object will become less and less dense.

8. But we see particles and things that are stable in the universe, how is your previous answer correct?

Answer: The force of gravity controls and limits the release of energy. It conserves energy. Gravity decides whether energy should be transferredlreleased or not. For example: If one lives near a black hole, then time for him will be slow than the rest of the cosmos. This happens because the transformations that occur in the body are less, the changes are less. But in empty space things work the opposite due to dark energy.

9. What happens when energy is no longer released?

Answer: Time will stop. No changes will take place.

10. How will such an object look alike?

Answer: Like a black hole (invisible) without gravity effects.

11. Is there a place in the universe where time stops?

Answer: Inside black holes, because inside black holes there is no force and motion. Another model: A universe with only photons will have no time, not in the sense time will stop, but time will flow infinitely, until gravity or something interferes.

12. Is it possible to time travel, visit the past?

Answer: No. But if there is a way to reverse all events then yes. But when one reverses events information will be destroyed (along with them) so one can only reverse time, not travel to the past.

The Original paper 'observations on time 2012' and this 'remade paper' provide logical answers to the following problems: [1] Fundamental question: what causes force, motion and time? [2] Entropy question: Arrow of time, [3] Unification of thermodynamics and general relativity; and [4] New insight to space and dark energy. BUT Note: This is only a fragment in the big picture.

\section{Acknowledgement}

Without the wonderful teachings of Benjamin Schumacher, Neil DeGrasse Tyson, Sean Carroll, Steven Pollock, and Alexei filippenko -I could not have understood these fundamental concepts. Special thanks to my parents, the teaching company: the great courses and to my late dog, Kutty.

Journal Paper:

\section{References:}

[1] SaiPrahlad K."Observations on time 2012”, IOSR Journal of Applied Physics (IOSRJAP) ISSN: 2278-4861 Volume 1, Issue 3 (July-Aug. 2012), PP 45-48

[2] A. Einstein, "Über das Relativitätsprinzip und die ausdemselbengezogenenFolgerungen", Jahrbuch der Radioaktivität und Elektronik 4, 411-462 (1907); English translation, in "On the relativity principle and the conclusions drawn from it", in "The Collected Papers", v.2, $433-484$ (1989); also in H M Schwartz, "Einstein's comprehensive 1907 essay on relativity, part I", American Journal of Physics vol.45,no.6 (1977) pp.512-517; Part II in American Journal of Physics vol.45 no.9 (1977), pp.811817; Part III in American Journal of Physics vol.45 no.10 (1977), pp.899-902, see parts I, II and III.

[3] Lorenzo Maccone. "Quantum solution to the Arrow-of-Time dilemma. ”Physical Review Letters 103, 080401 (2009).

\section{Books:}

[4] Planck, M. (1914). the theory of heat radiation. Masius, M. (transl.) (2nd ed.). P. Blakiston's Son \& Co. OL7154661M.

[5] Anne Rooney, 1001 Shocking Science Facts (Published 2008) ISBN: 978-1-84858-008-4

[6] Stephen Hawking, a brief history of time: from the big bang to black holes(Published 1988) ISBN: 978-0-553-10953-5

[7] "Eye, human"Encyclopædia Britannica. Encyclopedia Britannica Ultimate Reference Suite. Chicago: Encyclopædia Britannica, 2010.

[8] Carnot, Sadi,reflections on the motive power of fire (New York: Dover, 1960)

[9] Gardner, Martin, time travel and other mathematical bewilderments (New York: W. H. Freeman, 1988)

[10] Gott, J. Richard,time travel in Einstein's universe: the physical possibilities of travel through Time (New York: Houghton Mifflin, 2001)

[11] Kaku, Michio,physics of the Impossible: a scientific exploration into the world of phases, force fields, teleportation, and time travel (New York: Doubleday, 2008)

[12] Leff, Harvey S. and Andrew F. Rex, eds. Maxwell's demon 2: entropy, classical and quantum Information, computing (Bristol, UK: Institute of Physics, 2003)

[13] Polkinghorne, J. the quantum world (New York: Longman, 1984)

[14] Taylor, Edwin F. and John A. Wheeler.spacetime physics (New York: W. H. Freeman, 1992)

[15] Thorne, Kip S. black holes and time warps: Einstein's Outrageous Legacy (New York: W. W. Norton, 1994)

[16] Van Ness, H. C. understanding thermodynamics (New York: Dover, 1969)

[17] Wheeler, John A. a journey into gravity and spacetime (New York: Scientific American Library, 1990)

[18] Carroll, Sean, from eternity to here (Published 2010) ISBN: 0-525-95133-4

[19] Feynman, Richard Phillips, the character of physical law (Cambridge: MIT Press, 1965)

Science Magazine:

[20] Catchpole, Heather (200-09-04). Cosmos Online - Verging on absolute zero

Referral persons:1. Benjamin Schumacher, 2.Lorenzo Maccone, 3. Neil deGrasse Tyson, 4. Sean Carroll, 5.Michio Kaku. 